\title{
Pulse Shape Filtering in Wireless Communication-A Critical Analysis
}

\author{
A. S Kang \\ Assistant Professor, Deptt of Electronics and \\ Communication Engg \\ SSG Panjab University Regional Centre \\ Hoshiarpur, Punjab, INDIA \\ askang_85@yahoo.co.in
}

\author{
Vishal Sharma \\ Assistant Professor, Deptt of Electronics and \\ Communication Engg \\ University Institute of Engineering and Technology \\ Panjab University Chandigarh - 160014, INDIA \\ vishaluiet@yahoo.co.in
}

\begin{abstract}
The goal for the Third Generation (3G) of mobile communications system is to seamlessly integrate a wide variety of communication services. The rapidly increasing popularity of mobile radio services has created a series of technological challenges. One of this is the need for power and spectrally efficient modulation schemes to meet the spectral requirements of mobile communications. Pulse shaping plays a crucial role in spectral shaping in the modern wireless communication to reduce the spectral bandwidth. Pulse shaping is a spectral processing technique by which fractional out of band power is reduced for low cost, reliable, power and spectrally efficient mobile radio communication systems. It is clear that the pulse shaping filter not only reduces inter-symbol interference (ISI), but it also reduces adjacent channel interference. The present paper deals with critical analysis of pulse shaping in wireless communication.
\end{abstract}

Keywords- WCDMA, Pulse Shaping;

\section{INTRODUCTION}

Third generation cellular systems are being designed to support wideband services like high speed Internet access, video and high quality image transmission with the same quality as the fixed networks. Research efforts have been underway for more than a decade to introduce multimedia capabilities into mobile communications. Different standard agencies and governing bodies are trying to integrate a wide variety of proposals for third generation cellular systems. [1-7]

One of the most promising approaches to $3 \mathrm{G}$ is to combine a Wideband CDMA (WCDMA) air interface with the fixed network of GSM. Several proposal supporting WCDMA were submitted to the International Telecommunication Union (ITU) and its International Mobile Telecommunications for the year 2000 (IMT2000) initiative for 3G. All these schemes try to take advantage of the WCDMA radio techniques without ignoring the numerous advantages of the already existing GSM networks. The standard that has emerged is based on ETSI's Universal Mobile Telecommunication System (UMTS) and is commonly known as UMTS Terrestrial Radio Access (UTRA) [1]. The access scheme for UTRA is Direct Sequence Code Division Multiple Access (DSCDMA). The information is spread over a band of approximately $5 \mathrm{MHz}$. This wide bandwidth has given rise to the name Wideband CDMA or WCDMA.[8-9]

The future mobile systems should support multimedia services. WCDMA systems have higher capacity, better properties for combating multipath fading, and greater flexibility in providing multimedia services with defferent transmission rate and different QoS requirements and has been investigate worldwide [4-6]. CDMA mobile systems are interference limited and therefore reducing interference can directly increase system capacity.

\section{WIDEBAND CDMA}

In WCDMA, the CDMA technology (Code Division Multiple Access) air interface is implemented along with GSM networks. WCDMA is a Third Generation technology and works towards the interoperability between the various $3 \mathrm{G}$ technologies and networks being developed worldwide. WCDMA transmits on a $5 \mathrm{MHz}$ wide radio channel and hence is called Wideband CDMA. This $5 \mathrm{MHz}$ wide radio channel is in contrast to CDMA which transmits in one or more pairs of 1.25 $\mathrm{MHz}$ wide radio channel. WCDMA uses Direct Sequence spreading, where spreading process is done by directly combining the baseband information to high chip rate binary code. The Spreading Factor is the ratio of the chips (UMTS $=3.84 \mathrm{Mchips} / \mathrm{s}$ ) to baseband information rate. Spreading factors vary from 4 to 512 in FDD UMTS. Spreading process gain can in expressed in dBs (Spreading factor 128=21dBgain).

Figure 1.shows the CDMA spreading 


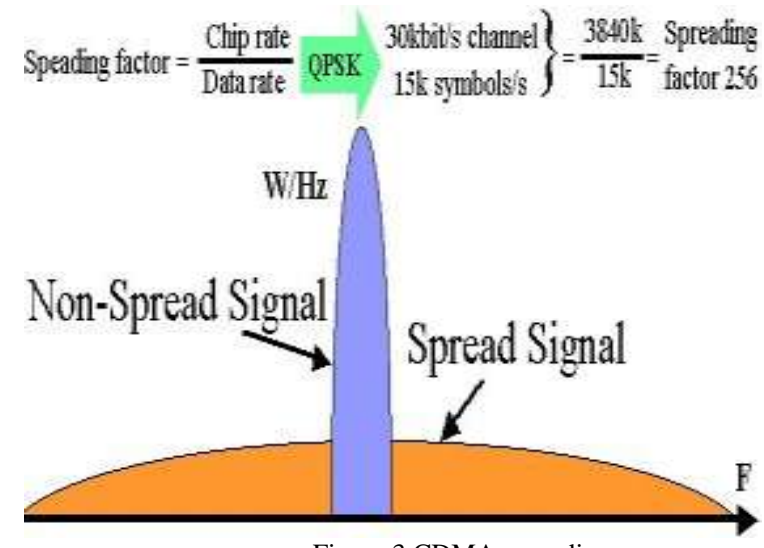

Figure 3.CDMA spreading

\section{A. Pulse Shape filtering in Wireless Communication}

Linear modulation methods such as QAM,QPSK, OQPSK have received much attention to their inherent high spectral efficiency However for the efficient amplification of transmitted signal, the Radio Frequency Amplifier is normally operated near the saturation region and therefore exhibit non linear behavior. As a result significant spectral spreading occurs, when a signal with large envelope variations propagates through such an amplifier and creates large envelope fluctuations.

To satisfy the ever increasing demands for higher data rates as well as to allow more users to simultaneously access the network, interest has peaked in what has come to be known as wideband code division multiple access (WCDMA).The basic characteristics of WCDMA waveforms that make them attractive for high data rate transmissions are their advantages over other wireless systems. It emphasizes that how the choice of spread bandwidth affects the bit error rate of system [10]

\section{Multipath EFFECTS}

Multipath propagation through linear dispersive media introduces distortion in signal during the wireless transmission. Due to this, there is degradation in BER performance, unless it is compensated with some suitable techniques at the receiver. In addition to frequency selectivity, the wireless channel also experiences time variations, which arise due to relative motion between transmitter and receiver which in turn needs to acquire mobile channel states and needs to be optimized. In a digital communication system, digital information can be sent on a carrier through changes in its fundamental characteristics such as: phase, frequency, and amplitude. In a physical channel, these transitions can be smoothed, depending on the filters implemented in transmission. In fact, the use of a filter plays an important part in a communications channel because it is effective at eliminating spectral leakage, reducing channel width, and eliminating interference from adjacent symbols (Inter Symbol Interference, ISI)[1-9].Transmitting a signal at high modulation rate through a band-limited channel can create intersymbol interference. As the modulation rate increases, the signal's bandwidth increases. When the signal's bandwidth becomes larger than the channel bandwidth, the channel starts to introduce distortion to the signal. This distortion is usually seen as intersymbol interference. The signal's spectrum is determined by the pulse shaping filter used by the transmitter. Usually the transmitted symbols are represented as a time sequence of dirac delta pulses.

\section{NeEd of EfFicient Pulse Shaping}

In communications systems, two important requirements of a wireless communications channel demand the use of a pulse shaping filter. These requirements are:

a) Generating band limited channels, and

b) Reducing Inter Symbol Interference (ISI) arising from multi-path signal reflections.

Both requirements can be accomplished by a pulse shaping filter which is applied to each symbol. In fact, the sinc pulse, shown below, meets both of these requirements because it efficiently utilizes the frequency domain to utilize a smaller portion of the frequency domain, and because of the windowing affect that it has on each symbol period of a modulated signal. A sinc pulse is shown below in figure 2 along with an FFT spectrum of the givensignal.

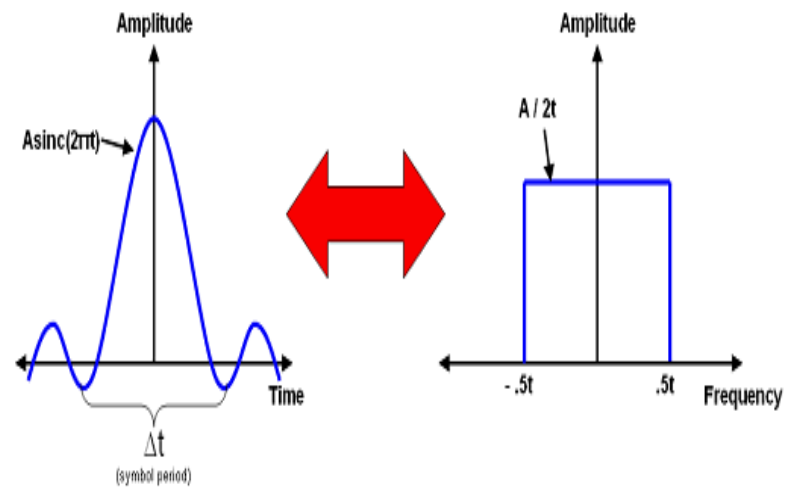

Figure 2 Time vs. Frequency Domain for a Sinc Pulse

The sinc pulse is periodic in nature and is has maximum amplitude in the middle of the symbol time. In addition, it appears as a square wave in the frequency domain and thus can effectively limit a communications channel to a specific frequency range. [10-12]

\section{A. Reducing Channel Bandwidth}

Fundamentally, modulation of a carrier sinusoid results in constant transitions in its phase and amplitude. Below, figure 3. shows the time domain of a carrier sinusoid with a symbol rate that is half of the carrier. It is clear that phase/amplitude transitions occur at every two periods of the carrier and sharp transitions occur, when filtering is not applied. [10-12] 


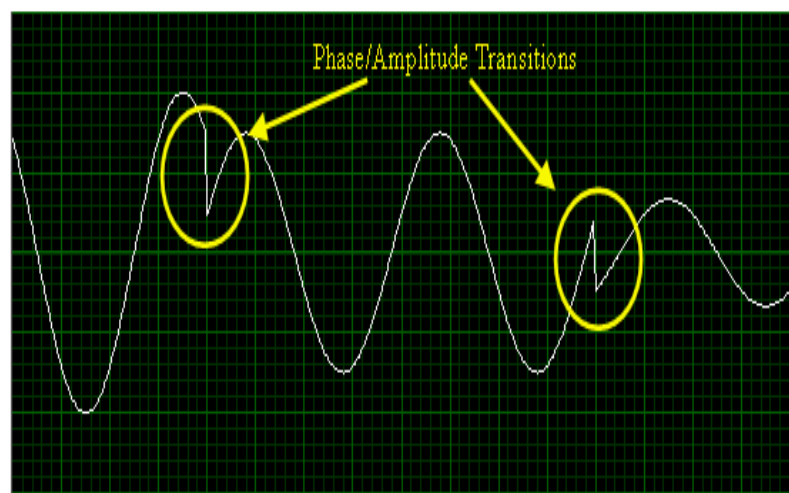

Figure 3. Phase and Amplitude Transitions in an Unfiltered Modulated Signal

The sharp transitions in any signal result in high-frequency components in the frequency domain. By applying a pulseshaping filter to the modulated sinusoid, the sharp transitions are smoothed and the resulting signal is limited to a specific frequency band. Below, it is shown time-domain modulated sinusoid.

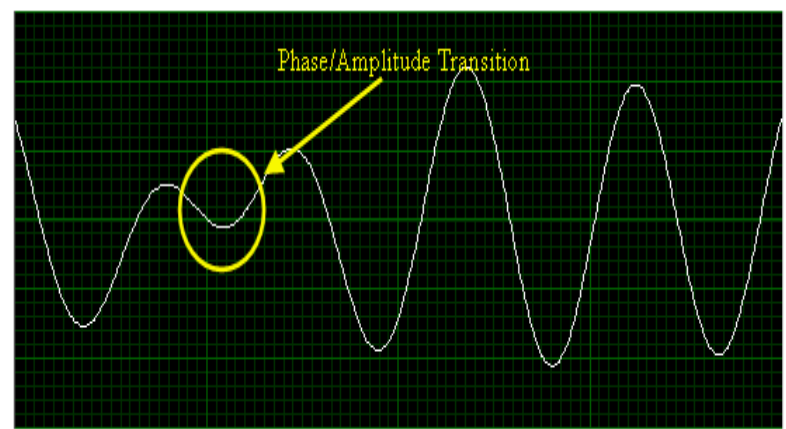

Figure 4. Smoothed Phase and Amplitude Transitions in a Filtered Modulated Signal

Fig 4 shows smoothed phase and amplitude transitions in a filtered modulated signal. It happens much more gradually when filtering is implemented. As a result, the frequency information of the sinusoid becomes more concentrated into a specified frequency band. [10]The sharp transitions do cause high frequency components in the frequency domain. Now, once a filter has been applied to the carrier signal, these high frequency components of the signal have been removed. Thus, the majority of the channel power is now limited to a specific defined bandwidth. It is clear that the required bandwidth for a channel is directly related to the symbol rate and is centered at the carrier frequency.

\section{B. Reducing Inter-Symbol Interference (ISI)}

In band limited channels, intersymbol interference (ISI) can be caused by multi-path fading as signals are transmitted over long distances and through various mediums. More specifically, this characteristic of the physical environment causes some symbols to be spread beyond their given time interval. As a result, they can interfere with the following or preceding transmitted symbols. One solution to this problem is the application of the pulse shaping filter. By applying this filter to each symbol that is generated, it is possible to reduce channel bandwidth while reducing ISI. In addition, it is common to apply a match filter on the receiver side to minimize these affects. Fig 5 shows the output in time domain. It is clear that the maximum amplitude of the pulse-shaping filter occurs in the middle of the symbol period. In addition, the beginning and ending portions of the symbol period are attenuated. Thus, ISI is reduced by providing a pseudo-guard interval which attenuates signals from multipathreflections[10].

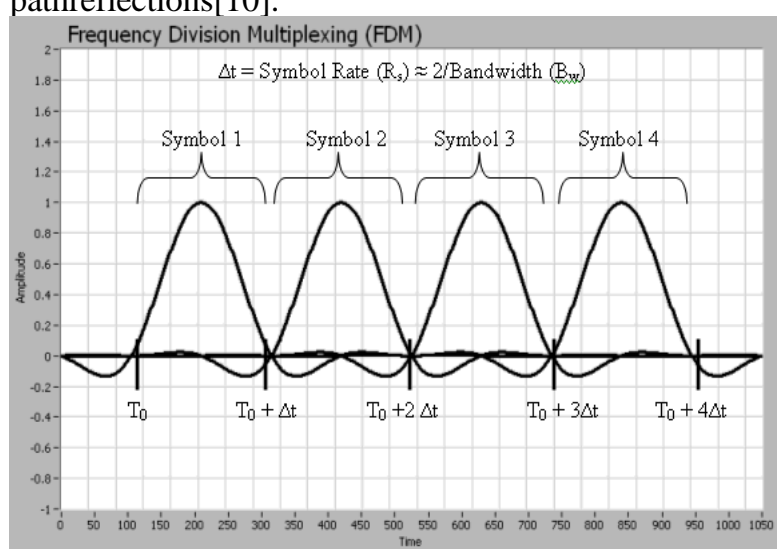

Figure 5 Filter Output in the Time Domain.

\section{Pulse Shaping and Matched Filtering}

The matched filter is perhaps equally as important as the pulse-shaping filter. While the pulse shaping filter serves the purpose of generating signals such that each symbol period does not overlap, the matched filter is important to filter out what signal reflections do occur in the transmission process. Because a direct-path signal arrives at the receiver before a reflected signal does, it is possible for the reflected signal to overlap with a subsequent symbol period. This is shown in the fig 6. It is clear, the matched filter reduces this affect by attenuating the beginning and ending of each symbol period. Thus, it is able to reduce intersymbol interference. One of the most common choices for a matched filter is the root raised cosinefilter[10].

Power

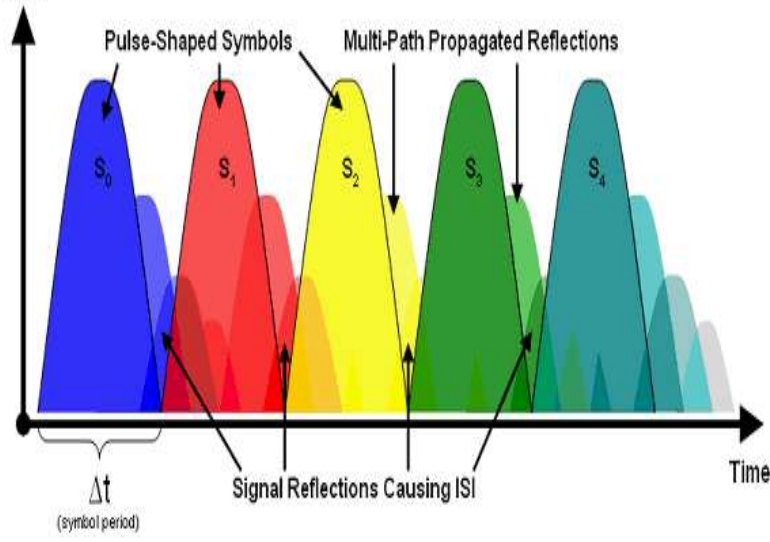

Figure 6. ISI Caused by Multi-Path Distortion

\section{DIFFERENT PULSE SHAPES}

1) Rectangular Pulse

2) Raised Cosine Pulse

3) Square Root Raised Cosine Pulse

4) Gaussian Pulse 
5) Flipped Exponential Pulse

6) Flipped Hyperbolic Secant Pulse

7) Flipped Inverse Hyperbolic Secant Pulse

Data transmission systems that must operate in a bandwidth-limited environment must contend with the fact that constraining the bandwidth of the transmitted signal necessarily increases the likelihood of a decoding error at the receiver. Bandwidth limited systems often employ pulse-shaping techniques that allow for bandwidth containment while minimizing the likelihood of errors at the receiver.[12]

\section{A. Rectangular Pulse}

The most basic information unit in a digital transmission scheme is a rectangular pulse. It has a defined amplitude, A, and defined duration, T. Such a pulse is shown in Figure 7, where $\mathrm{A}=1, \mathrm{~T}=\mathrm{To}$, with the pulse centered about the time origin at $\mathrm{t}=0$. Typically, a sequence of such pulses (each delayed by $\mathrm{T}$ seconds relative to the previous one) constitutes the transmission of information. The information, in this case, is encoded in the amplitude of the pulse. The simplest case is when a binary 0 is encoded as the absence of a pulse $(A=0)$ and a binary 1 is encoded as the presence of a pulse $(\mathrm{A}=$ constant). Since each pulse spans the period $\mathrm{T}$, the maximum pulse rate is $1 / \mathrm{T}$ pulses per second, which leads to a data transmission rate of $1 / \mathrm{T}$ bits per second.[12]

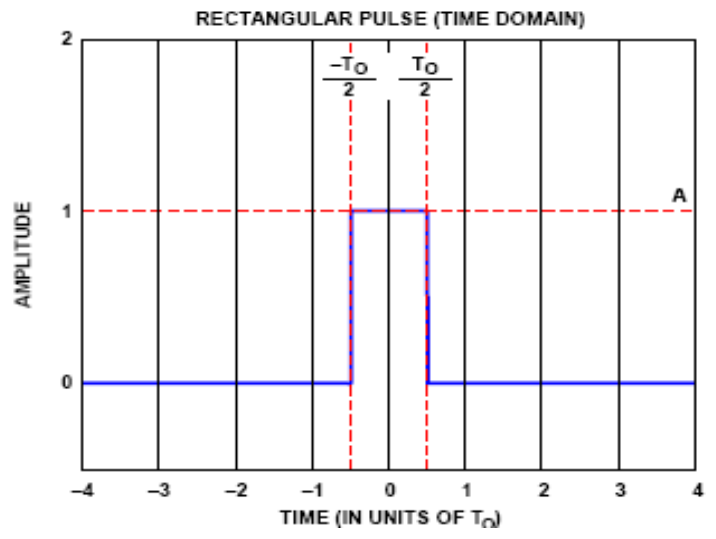

Figure 7. A Single Rectangular Pulse $\left(T=T_{0}, A=1\right)$

The pulses used to transmit symbols occupy a fixed time interval, $\mathrm{T}$.Thus, the pulse rate is $1 / \mathrm{T}$ pulses per second, which leads to a symbol rate of $1 / \mathrm{T}$ symbols per second. The unit, symbols per second, is often referred to as baud. The data transmission rate in bits per second is the baud rate multiplied by the number of bits represented by each symbol. For example, if a symbol represents four bits, then the bit rate is four times the symbol rate. This means that a lower transmission rate can be used to transmit symbols as opposed to directly transmitting bits, which is the primary reason that the more sophisticated data transmission systems encode groups of bits into symbols. The logic 1 is represented by the presence of a pulse of unit amplitude and a logic 0 by the absence of a pulse (that is, zero amplitude).

\section{B. Raised Cosine Pulse}

As shown in Figure 3, the spectrum of a rectangular pulse spans infinite frequency. In many data transmission applications, the transmitted signal must be restricted to a certain bandwidth. This can be due to system design constraints In such instances, the infinite bandwidth associated with a rectangular pulse is not acceptable. The bandwidth of the rectangular pulse can be limited, however, by forcing it to pass through a low-pass filter. The act of filtering the pulse causes its shape to change from purely rectangular to a smooth contour without sharp edges.[12] Therefore, the act of filtering rectangular data pulses is often referred to as pulse shaping. Unfortunately, limiting the bandwidth of the rectangular pulse necessarily introduces a damped oscillation. That is, the rectangular pulse exhibits nonzero amplitude only during the pulse interval, whereas the smoothed (or filtered) pulse exhibits ripples both before and after the pulse interval. At the receiver, the ripples can lead to incorrect decoding of the data, because the ripples associated with one pulse interfere with the pulses before and after it. However, the choice of a proper filter can yield the desired bandwidth reduction while maintaining a time domain shape that does not interfere with the decoding process of the receiver.[12]

This filter is the well-known raised cosine filter and its frequency response is given by

$$
\begin{aligned}
& \mathrm{H}(\mathrm{w})=\tau \ldots \ldots \ldots \ldots \ldots \ldots \ldots \ldots \ldots, \mathrm{w} \leq \mathrm{c} \\
& \tau\left\{\cos ^{2}[\tau(\mathrm{w}-\mathrm{c}) / 4 \alpha]\right\} \ldots \ldots \ldots \ldots \mathrm{c} \leq \mathrm{w} \leq \mathrm{d}
\end{aligned}
$$

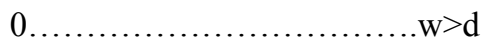

where $\mathrm{w}$ is the radian frequency $2 \pi \mathrm{f}$,

$\tau$ is the pulse period

$\alpha$ is roll off factor

$\mathrm{c}$ is equal to $\pi(1-\alpha) / \tau$

$\mathrm{d}$ is equal to $\pi(1+\alpha) / \tau$

A plot of the raised cosine frequency response is shown in Figure 8 (normalized to $\tau=1$ ). The raised cosine filter gets its name from the shape of its frequency response, rather than its impulse (or time domain) response.

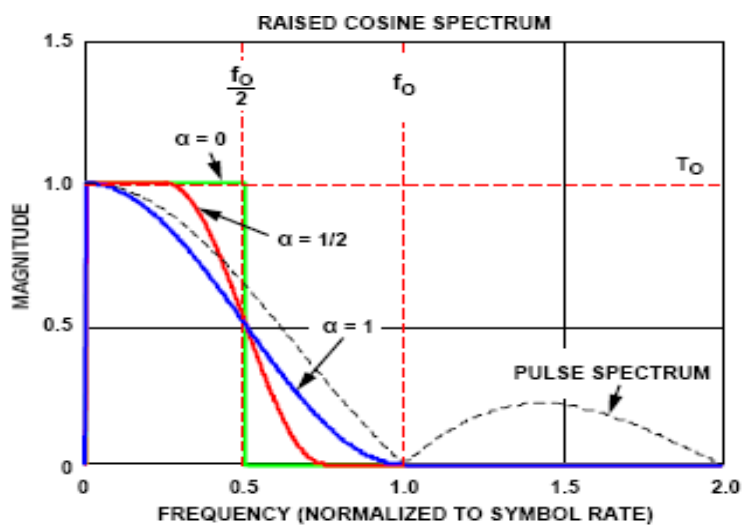

Figure 8. The Raised Cosine Frequency Response

Ideal Response of Raised Cosine Filter is shown in figure 9. below[10] 

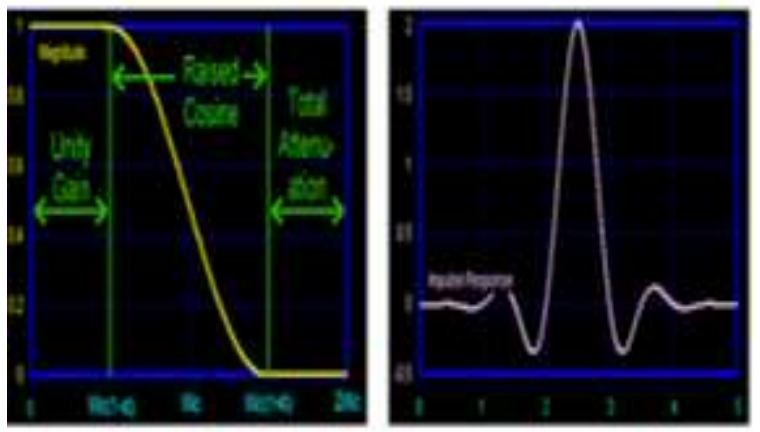

Figure 9. Ideal Response of Raised Cosine Filter

Ideal Raised Cosine filter frequency response consists of unity gain at low frequencies, raised cosine function in the middle and total attenuation at high frequencies. The root raised cosine filter is generally used in series pairs so that total filtering effect is that of raised cosine filter.Sometimes it is desirable to implement the raised cosine response as the product of two identical responses, one at the transmitter and the other at the receiver. In such cases, the response becomes a square-root raised cosine response since the product of the two responses yields the desired raised cosine response.[12]

\section{Square Root Raised Cosine}

The frequency Response of the Square-Root Raised Cosine is given as below.

$$
\begin{aligned}
& H(w)=\sqrt{ } \tau \ldots \ldots \ldots \ldots \ldots \ldots \ldots \ldots \ldots \ldots \ldots \ldots \\
& \sqrt{ } \tau\{\cos [\tau(\mathrm{w}-\mathrm{c}) / 4 \alpha]\} \ldots \ldots \ldots . \mathrm{c} \leq \mathrm{w} \leq \mathrm{d}
\end{aligned}
$$

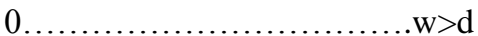

The variable definitions are the same as for the raised cosine response.The consequence of pulse shaping is that it distorts the shape of the original time domain rectangular pulse into a smoothly rounded pulse with damped oscillations (ripples) before and after the $\pm 1 / 2$ To points. The ripples result from the convolution of the rectangular pulse with the raised cosine impulse response (convolution is the process of filtering in the time domain). This is a source of decision-making error at the receiver known as Intersymbol Interference (ISI). Reduced bandwidth means larger ripple, which exacerbates ISI and increases the likelihood of an incorrect decision (that is, error) at the receiver.[12] Obviously, a trade off exists between bandwidth containment in the frequency domain and ripple attenuation in the time domain. It is this trade off of bandwidth containment vs. ripple amplitude that must be considered by design engineers when developing a data transmission system that employs pulse shaping.Ideal response of Square Root Raised Cosine filter is shown in figure 10 below.[10]

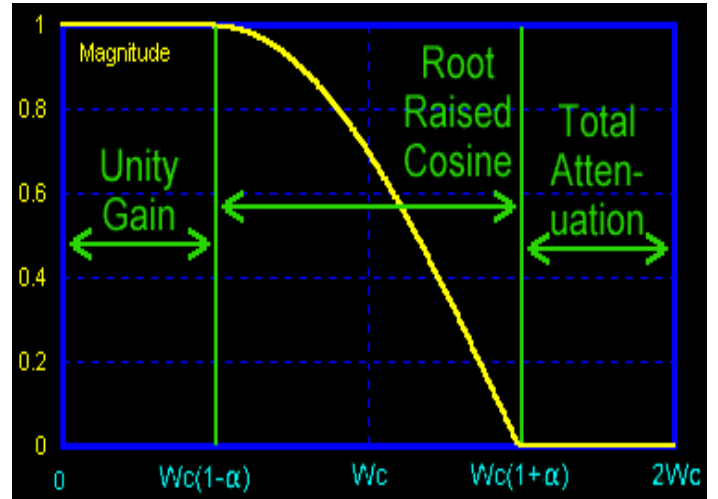

Figure 10. Ideal Square Root Raised Cosine Filter frequency Response

\section{Gaussian Pulse}

This gives an output pulse shaped like a Gaussian function. The Gaussian filter is a pulse shaping technique that is typically used for Frequency Shift Keying (FSK) and Minimum Shift Keying (MSK) modulation. This filter is unlike the raised cosine and root raised cosine filters because it does not implement zero crossing points. The impulse response for the Gaussian filter is defined bythefollowingequation: $h_{\mathrm{G}}(t)=Q\left(\frac{2 \pi \alpha}{\sqrt{\ln 2}}\left(n-\frac{1}{2}\right)\right)-Q\left(\frac{2 \pi \alpha}{\sqrt{\ln 2}}\left(n+\frac{1}{2}\right)\right)$

Graphical representation of the impulse response of gaussian filter is shown in figure11. As described above, it is clear that there are no zero crossings for this type of filter[10].

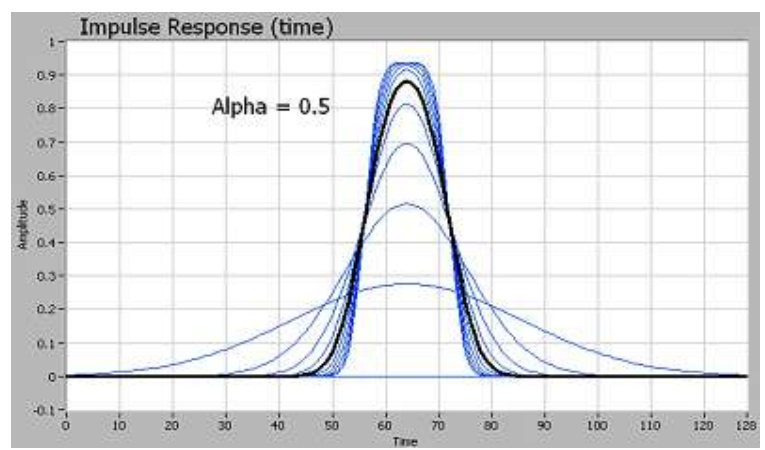

Figure 11. Impulse Response of Gaussian Filter

\section{E. Flipped Exponential Pulse}

This pulse is proposed by Beaulieu(fexp)[11]. It is known as flipped-exponential pulse.In this pulse a new parameter, $\beta=\ln$ $2 / \alpha \beta$ has been introduced .The frequency and impulse responses of this family are given as below. 
$\mathrm{S} 2(\mathrm{f})=1 \ldots \ldots \ldots \ldots \ldots . \mathrm{f} \leq \mathrm{B}(1-\alpha)$;

$\mathrm{S} 2(\mathrm{f})=\exp (\beta(\mathrm{B}(1-\alpha)-\mathrm{f})) \ldots \ldots \ldots \ldots . \mathrm{B}(1-\alpha)<\mathrm{f} \leq \mathrm{B}$

$\mathrm{S} 2(\mathrm{f})=1-\exp (\beta(\mathrm{f}-\mathrm{B}(1+\alpha))) \ldots \ldots \ldots . \mathrm{B}<\mathrm{f} \leq \mathrm{B}(1+\alpha)$

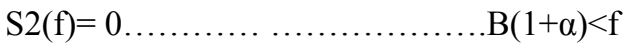

$\mathrm{S} 2(\mathrm{t})=(1 / \mathrm{T}) \operatorname{sinc}(\mathrm{t} / \mathrm{T})\left(4 \beta \pi \mathrm{t} \sin (\pi \alpha \mathrm{t} / \mathrm{T})+2 \beta^{2} \cos (\pi \alpha \mathrm{t} / \mathrm{T})-\right.$

$\left.\beta^{2}\right) /\left[(2 \pi \mathrm{t})^{2}+\beta^{2}\right]$

Two more pulses have been derived from from flipped exponential pulse[11]

\section{F. Flipped Secant Hyperbolic Pulse}

The first one is known as flipped-hyperbolic secant (fsech) pulse, has frequency and impulse responses defined as $\mathrm{S} 3(\mathrm{f})=1 \ldots \ldots \ldots \ldots \ldots \ldots . \mathrm{f} \leq \mathrm{B}(1-\alpha)$;

$$
\begin{aligned}
& =\operatorname{sech}(\gamma|\mathrm{f}|-\mathrm{B}(1-\alpha))) \ldots \ldots \mathrm{B}(1-\alpha)<\mathrm{f} \leq \mathrm{B} \\
& =1-\operatorname{sech}(\gamma(\mathrm{B}(1+\alpha)-\mathrm{f})) \ldots . \mathrm{B}<\mathrm{f}<\leq \mathrm{B}(1+\alpha) \\
& =0 \ldots \ldots \ldots \ldots \ldots \ldots \ldots \ldots . \mathrm{B}(1+\alpha)<\mathrm{f}
\end{aligned}
$$

$\mathrm{S} 3(\mathrm{t})=(1 / \mathrm{T}) \operatorname{sinc}(\mathrm{t} / \mathrm{T})\left\{8 \pi \mathrm{t} \sin (\pi \alpha \mathrm{t} / \mathrm{T}) \mathrm{F}_{1}(\mathrm{t})+2 \cos (\pi \alpha \mathrm{t} / \mathrm{T})[1-\right.$

$\left.\left.2 \mathrm{~F}_{2}(\mathrm{t})\right]+4 \mathrm{~F}_{3}(\mathrm{t})-1\right\}$

where $\gamma=\ln (\sqrt{3}+2) / \alpha \beta$

The impulse response has been obtained through an expansion in series of exponentials.

\section{G. Flipped Inverse Secant Hyperbolic Pulse}

The second pulse is referred to as flipped-inverse hyperbolic secant (farcsech) pulse. It has frequency response defined as

$$
\begin{aligned}
& \mathrm{S} 4(\mathrm{f})=1 \ldots \ldots \ldots \ldots \ldots \ldots \ldots \ldots \ldots \ldots \ldots \ldots \ldots \ldots \ldots \ldots, \mathrm{f}(1-\alpha) \\
& =1-(1 / 2 \alpha \beta \gamma) \operatorname{arcsech}(1 / 2 \alpha \beta(B(1+\alpha)-f)) \ldots \ldots \ldots . . B(1- \\
& \alpha)<\text { f } \leq \text { B } \\
& =(1 / 2 \alpha \beta \gamma) \operatorname{arcsech}(1 / 2 \alpha \beta(\mathrm{f}-\mathrm{B}(1- \\
& \alpha))) \ldots \ldots \ldots \ldots . \mathrm{B}<\mathrm{f} \leq \mathrm{B}(1+\alpha)
\end{aligned}
$$

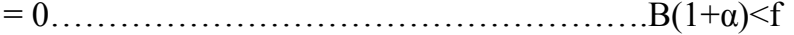

Its impulse response $\mathrm{s} 4(\mathrm{t}) \mathrm{can}$ be evaluated through a numerical inverse Fourier transform. The plot of frequency responses of the above pulses is shown in figure 12 below. It is clear that the pulses are real and even, with optimum time sampling.

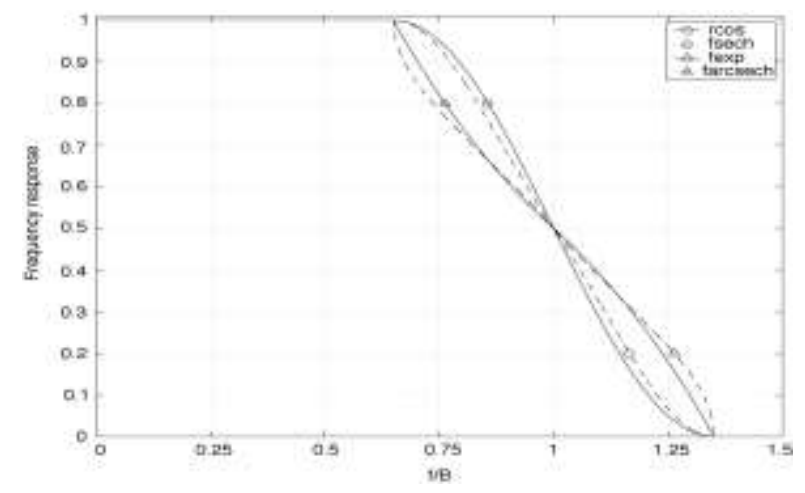

Figure 12. Frequency Responses of the different pulses with roll-off factor $\alpha=$ 0.35

\section{CONCLUSION}

Digital Signal processing techniques are being used to improve the performance of $3 \mathrm{G}$ systems WCDMA (Wideband Code-Division Multiple Access), an ITU standard derived from Code-Division Multiple Access (CDMA), is officially known as IMT-2000 direct spread spectrum. W-CDMA is a thirdgeneration $(3 \mathrm{G})$ mobile wireless technology that promises much higher data speeds to mobile and portable wireless devices than commonly offered in today's market. The pulse shaping filter plays a critical role in WCDMA performance enhancement. [13-20]. The present paper has critically analyzed the pulse shape filtering in wireless communication. The application of signal processing techniques to wireless communications is an emerging area that has recently achieved dramatic improvement in results and holds the potential for even greater results in the future as an increasing number of researchers from the signal processing and communication areas participate in this expanding field.

\section{IMPACT OF STUDY}

1. The study is useful to improve the performance of WCDMA Network.

2. In the planning of WCDMA Network.

3. To achieve the flexibility in use of data rates in different environments.

4. Design of future cellular mobile communication network.

\section{FUTURE SCOPE OF WORK}

The following points have to be concentrated to extend its application to wide range of future directions:

1. Different multipath fading channels can replace AWGN channel in the model to simulate the system under different mobile radio channels.

2. More influencing parameters can be incorporated in the simulation model using adaptive signal processing.

3. The simulation model can be developed for Tan, Beaulieu and Damen pulse shaping families by incorporating more variables.

4. DSP algorithms can be developed for performance enhancement of WCDMA based wireless system using optimized values of parameters of pulse shaping filters.

5. Simulation study can be extended to different data rates such as $144 \mathrm{kbps}, 384 \mathrm{kbps} \& 2 \mathrm{Mbps}$.

\section{ACKNOWLEDGEMENT}

The first author is thankful to Dr B S Sohi, Director UIET,Sec-25,PANJAB UNIVERSITY Chandigarh for discussion and valuable suggestions during technical discussion at WOC conference at Panjab Engg College Chandigarh during presentation of research paper,'Digital Processing and Analysis of Pulse Shaping Filter for wireless communication "The help rendered by $\mathrm{S}$ Harjitpal Singh,a 
research scholar of NIT Jalandhar as well as Mr Hardeep Singh,Research Scholar, Communication Signal Processing research Lab, Deptt of Electronics Technology GNDU Amritsar is also acknowledged.

\section{REFERENCES}

[1] R.M Piedra and A. Frish, "Digital Signal Processing comes of age" IEEE spectrum, vol 33,No.5,pp70,May 1996.

[2] J Stevens, ,'DSPs in Communication "IEEE Spectrum vol.35,No.9,pp3946,Sept.98.

[3] C. Iniaco and D. Embres," "The DSP decision-fixed or floating?" IEEE Spectrum, Vol.3,No.9,pp 72-74,1996.

[4] P.M. Grant, "Signal Processing Hardware and Software" IEEE signal Processing Magazine,vol13,No.1,pp 86-92,Jan 1992.

[5] G. Giannkis, "Highlights of Signal Processing for communications" IEEE Signal Processing Magazine, vol 16,No.2,pp 14-51,March,1999.

[6] F.Adachi,"Wireless Past and Future-Evolving Mobile Communication Systems,"IEICE Trans $\quad$ Fundamentals,Vol.E84-A,No.1,pp.5560,Jan2001.

[7] H. Holma and A. Toskala, "WCDMA for UMTS, "John Wiley \&Sons.Ltd 2002.

[8] www.3GPP.org

[9] Tero Ojanpera and Ramjee Prasad, "Wideband CDMA for Third Generation Mobile Communications," Artech House, Boston, London, 1998 .

[10] National Instrument Signal Generator tutorial, Pulse shaping to improve spectral efficiency. www.ni.com/rf.

[11] Antonio Assalini,Andrea M.Tonello,"Improved Nyquist Pulses",IEEE Communication letters, vol 8 No 2, pp no 87-89, Feb2004.

[12] Ken Gentile,"The care and feeding of digital pulse shaping filters"www.rfdesign.com,pp-50-61, 2002.

[13] ASKang,ErVishalSharma“"Comparatative Performance Analysis of Pulse Shaping Techniques for Wireless Communication"proceedings of WECON -2008,International Conference on Wireless networks and Embedded Systems"(presesnted)Chitkara Institute of Engg \& Technology,Rajpura,Distt Patiala, pp 113-115,2008, Oct2008.
[14] ASKang,ErVishalSharma"Digital Signal Processing For Cellular Wireless Communication Systems presented at IEEE-ISM 2008,Indian Institute of Science,Bangalore(INDIA),pp-323-327, 3Dec-6Dec2008.

[15] A S Kang ,Er Vishal Sharma "Digital Processing and analysis of pulse shaping Filter for wireless Communication",presented at $2^{\text {nd }}$ National Conference(Co -Sponsered by IEEE, Chandigarh Sub Section) On Wireless and Optical Communication WOC-2008 at PEC Chandigarh(INDIA) pp110-113, 18-19 Dec,2008.

[16] A S Kang,Er Vishal Sharma "Pulse Shaping Filters in Wireless Communication-A Critical Review"Proceedings of National conference on optical and wireless communication(NCOW-2008, Co-Sponsered by Institution of Engineers(IE), INDIA) DAVIET,Jalandhar(INDIA) ,pp 291-297, November 27-28, 2008.

[17] A S Kang,Vishal Sharma "Spectral analysis of filters in celluilar wireless communication systems", International Conference on Systemics,Cybernetics,Informatics,ICSCI-2009,Hyderabad,pp-48-55, January7-10,2009.

[18] AS Kang,Vishal Sharma IEEE -0196,"Study of Spectral Analysis of Filters in Cellular Communication Systems" by A S Kang, Vishal Sharma in IEEE International advance Computing Conference (IACC09)held at Thapar University ,Patiala,(presented),pp 19341938, 6-7March 2009.

[19] ASKang,VishalSharmaIEEE0197,"Simulative Investigation of Pulse shaping in WCDMA Wireless Communication" by A S Kang, Vishal Sharma in IEEE International Advance Computing Conference(IACC09) held at Thapar University ,Patiala(presented),pp 1939-1943,6-7 March2009.

[20] ASKang,Vishal Sharma "Study of pulse shaping fiters in WCDMA under different interferences",IEEE-SIBCON2009(IEEE Siberian Conference on Control and Communications)March2009Russia,pp3741,28 March2009.

[21] Adibi, S. (2010). Traffic Classification - Packet- , Flow- , and Application-based Approaches. International Journal of Advanced Computer Science and Applications - IJACSA, 1, 6-15.

[22] Sharma, S. (2010). Efficient Implementation of Sample Rate Converter. International Journal of Advanced Computer Science and Applications - IJACSA, 1(6), 35-41. 